\title{
GENERALIZED WINTGEN INEQUALITY FOR LAGRANGIAN SUBMANIFOLDS IN QUATERNIONIC SPACE FORMS
}

\author{
Gabriel Macsim And VAlentin GhişoiU
}

Abstract. In this paper, we obtain the generalized Wintgen inequality, also known as the DDVV inequality, in the case of a Lagrangian submanifold in quaternionic space forms. We also give a proof for the DDVV inequality in the case of slant submanifolds of a quaternionic space form.

Mathematics subject classification (2010): 53C40, 53C25.

Keywords and phrases: Wintgen inequality, DDVV conjecture, Lagrangian submanifolds, slant submanifolds, quaternionic space forms.

\section{REFERENCES}

[1] T. CHOI AND Z. Lu, On the DDVV conjecture and the comass in calibrated geometry I, Math. Z. 260, (2008), 409-429.

[2] I. V. GuAdalupe AND L. RodRiguez, Normal curvature of surfaces in space forms, Pacific J. Math. 106, (1983), 95-103.

[3] S. Haesen and L. Verstraelen, Natural intrinsic geometrical symmetries, SIGMA Symmetry Integrability Geom. Methods Appl. 5, (2009), 15, Paper 086.

[4] Z. LU, Normal scalar curvature conjecture and its applications, J. Funct. Anal. 261, (2011), 1284 1308.

[5] A. MinaI, An inequality for totally real surfaces in complex space forms, Kragujevac J. Math. 26, (2004), 83-88.

[6] I. MinaI, On the generalized Wintgen inequality for Lagrangian submanifolds in complex space forms, Nonlinear Anal. 95, (2014), 714-720.

[7] B. RouxeL, Sur une famille des A-surfaces d'un espace Euclidien $\mathbb{E}^{4}$, Österreischer Mathematiker Kongress, Insbruck, (1981), p. 185.

[8] P. J. De Smet, F. Dillen, L. Verstraelen and L. VRancken, A pointwise inequality in submanifold theory, Arch. Math. (Brno), 35, (1999), 115-128.

[9] P. Wintgen, Sur l'inégalité de Chen-Willmore, C. R. Acad. Sci., Paris Sér. A-B, 288, (1979), A993A995. 